Abstracted/indexed in Academic Search Complete, Asia Journals Online, Bangladesh Journals Online, Biological Abstracts, BIOSIS Previews, CAB

Abstracts, Current Abstracts, Directory of Open Access Journals, EMBASE/Excerpta Medica, Google Scholar, HINARI (WHO), International

Pharmaceutical Abstracts, Open J-gate, Science Citation Index Expanded, SCOPUS and Social Sciences Citation Index;

\title{
Amyloid precursor protein in peripheral granulocytes as a potential biomarker for Alzheimer's disease
}

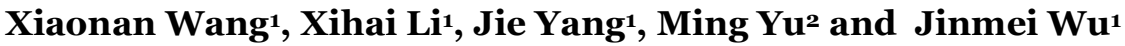 \\ ${ }^{1}$ College of Biotechnology, Jiangsu University of Science and Technology, Zhenjiang City, 212 018, China; \\ ${ }^{2}$ Department of Neurology, Affiliated Hospital of Jiangsu University, Zhenjiang City, 212001 , China.
}

\begin{tabular}{|c|c|}
\hline \multicolumn{2}{|l|}{ Article Info } \\
\hline $\begin{array}{l}\text { Received: } \\
\text { Accepted: } \\
\text { Available Online: }\end{array}$ & $\begin{array}{r}16 \text { January } 2016 \\
31 \text { January } 2016 \\
6 \text { March } 2016\end{array}$ \\
\hline \multicolumn{2}{|c|}{ DOI: 10.3329/bjp.v11iS1.26412 } \\
\hline \multicolumn{2}{|c|}{$\begin{array}{l}\text { Cite this article: } \\
\text { Wang X, Li X, Yang J, Yu M, Wu J. } \\
\text { Amyloid precursor protein in periph- } \\
\text { eral granulocytes as a potential bi- } \\
\text { omarker for Alzheimer's disease. } \\
\text { Bangladesh J Pharmacol. 2016; 11: S92 } \\
\text {-S97. }\end{array}$} \\
\hline
\end{tabular}

\begin{abstract}
The aim of this study was to assess the potential of amyloid precursor protein in peripheral granulocytes as a diagnostic biomarker for early detection of Alzheimer's disease. Immunohistochemistry and flow cytometry were used to evaluate amyloid precursor protein expression levels and subcellular localization in Alzheimer's disease. Much higher amyloid precursor protein expression was observed in some leukocytes from Alzheimer's disease patients, compared with samples from non-Alzheimer's disease controls. In addition, flow cytometry data indicated significantly higher amyloid precursor protein expression in granulocytes from Alzheimer's disease patients compared with control values. No statistically significant differences in amyloid precursor protein expression were obtained in lymphocytes or monocytes between the patient groups. In conclusion, amyloid precursor protein expression level in peripheral blood granulocyte is a potential biomarker for early diagnosis of Alzheimer's disease.
\end{abstract}

\section{Introduction}

Alzheimer's disease is a progressive neurodegenerative ailment, accounting for $60 \%$ of all dementia cases. Dementia affects 63 million people worldwide with the prevalence projected to reach 114 million by 2050 (Ratnavalli et al., 2002).

The pathogenesis of Alzheimer's disease is complex, and its etiology is not fully understood. Previous studies have suggested that the Alzheimer's disease onset process comprises of stages like pathogenesis without symptoms, mild cognitive impairment, and developing stage. Pathological features include: a) brain atrophy (Gustaw-Rothenberg et al., 2010); b) presence of multiple inflammatory plaques, i. e. senile plaques (Kawahara, 2004; Tabaton and Piccini, 2005), outside the

This paper was presented in the 3rd International Conference on Biomedicine and Pharmaceutics in Zhuhai, China, on December $11-13,2015$. cranial nerve cells; c) neurofibrillary tangles (Avila et al., 2004) in nerve cells; and d) decreased number of neurons and synapses (Tortosa et al., 1998). Molecular pathology changes appear several years or even decades before cognitive impairment in Alzheimer's disease patients. Therefore, finding a typical decisive pathogenic marker is the key to early Alzheimer's disease diagnosis (Gustaw-Rothenberg et al., 2010).

Amyloid precursor protein (APP) is a type I transmembrane protein, with a membrane receptor like structure. It is closely associated with Alzheimer's disease occurrence and development. In vivo, APP undergoes degradation mainly through two pathways. In the a-secretase pathway, APP is cleaved by a- and $\gamma$ secretases sequentially, producing secreted $\mathrm{APPa}$ (sAPPa), P3 (A $\beta 17-40$ or A $\beta 17-42)$, and the APP intracellular domain. As a-secretases cleave within the fragment precursor of the Alzheimer's disease-associated peptide amyloid beta, no full-length amyloid beta is formed. So, this pathway is considered part of the non- 
amyloidogenic pathway in APP processing. In the $\beta$ secretase pathway, also called amyloidogenic pathway, $\mathrm{APP}$ is sequentially cleaved by $\beta$ - and $\gamma$-secretases, producing extracellular APP (sAPP $\beta), A \beta(A \beta-40, A \beta-$ 42 ) and the APP intracellular domain. It is believed that the two degradation pathways co-exist, with $\alpha-$ and $\beta$ enzymes maintaining a relative balance and competing for the substrate. Under normal physiological conditions, the a-secretase pathway is predominant in APP processing, while in pathological conditions, the $\beta$ secretase pathway has the priority, producing $A \beta-40$ and $A \beta-42$ and forming senile plaques which cause Alzheimer's disease (Dulubova et al., 2004; Hardy and Selkoe, 2002). However, the specific mechanism regulating the two enzymes remains unclear.

Multiple studies have been carried out to identify Alzheimer's disease biomarkers. Interestingly, three biomarkers have been well-established and validated internationally for diagnosis in CSF samples by ELISA: $\mathrm{A} \beta$ (1-42), total tau, and phospho-tau-181. It is widely accepted that only the combination of these three CSF biomarkers significantly increases the diagnostic validity for sporadic Alzheimer's disease, with sensitivity and specificity of $>95 \%$ and $>85 \%$, respectively (Blennow 2005; Blennow et al., 2010; Marksteiner et al., 2007).

Peripheral blood samples are more convenient for diagnosis compared with other tissues such as brain tissues or CSF. Routine diagnosis of Alzheimer's disease and mixed forms of dementia from CSF has several drawbacks: Lumbar puncture and CSF collection is an invasive method with potential adverse effects; screening of patients is often difficult, and follow-up analysis over several years is problematic. Consequently, identifying useful Alzheimer's disease biomarkers from peripheral blood to provide insights on Alzheimer's disease diagnosis has gained increasing attention (Blennow, 2005).

Human platelets and lymphocytes produce all forms of APP and secrete amyloidogenic A $\beta$ peptides. These tissues may be useful in monitoring the responses to therapeutic interventions directed at APP metabolism (Li et al., 1999). Regarding APP in peripheral blood as a potential biomarker for the diagnosis of Alzheimer's disease, some interesting data have been reported (Padovani et al., 2002). In platelets, APP comprises three major forms with molecular weights of $130 \mathrm{kDa}, 110$ $\mathrm{kDa}$, and $106 \mathrm{kDa}$, respectively. The ratio of high molecular weight (130 kDa) APP form to that of low molecular weight $(110 \mathrm{kDa}$ and $106 \mathrm{kDa})$ is termed platelet APP form ratio (APPr). It has been shown that alteration of platelet APP processing is an early event in Alzheimer's disease, and APPr measurement might be of diagnostic value in differentiating mild Alzheimer's disease from normal aging (Borroni et al., 2002; Evin and Li, 2012; Mukaetova-Ladinska et al., 2012). Other researchers investigated APP-N expression in platelets, and found that APP-N levels were negatively correlated with cognitive scores (Mukaetova-Ladinska et al., 2012). Finally, a recent promising study showed that combination of 18 selected biomarkers (chemokines, cytokines, growth factors and binding proteins) in plasma might allow the diagnosis of Alzheimer's disease (Mangialasche et al., 2013) and MCI with nearly $90 \%$ accuracy.

Our preliminary data showed that APP expression in peripheral blood cells was increased in Alzheimer's disease patients compared to non-Alzheimer's disease controls (data not shown). The APP protein plays an important role in the pathogenesis of Alzheimer's disease, and APP changes appear very early within and outside the central nervous system in patients with Alzheimer's disease (Jiang et al., 2003). In addition, a recent study suggested that white blood cell counts can be used as peripheral markers for Alzheimer's disease (Shad et al., 2013). To further explore the effectiveness of peripheral blood cell APP as an Alzheimer's disease biomarker, we assessed the expression levels and subcellular localization of APP in peripheral blood cells by immunochemistry/immunofluorescence and flow cytometry techniques. Our findings indicate that APP expression in peripheral granulocytes is a potential biomarker for Alzheimer's disease, providing a basis for Alzheimer's disease prevention and cure.

\section{Materials and Methods \\ Cases}

Samples were obtained from a total of 18 individuals. All specimens were collected with informed consent and under IRB-approved protocols.

A total of 9 newly diagnosed Alzheimer's disease patients (4 males and 5 females; 53-87 years old) from Jiangbin Hospital (ZhenJiang City, Jiangsu Province) were randomly selected. Patients had no previous drug treatment and fulfilled the American NINCDSADRDA Alzheimer's criteria. Each patient underwent the minimum mental state examination which was scored. Combined with electroencephalography, CT, MRI or SPECT, patients with other types of dementia were excluded.

The control group comprised randomly selected healthy people (males 4; females 5) at the Jiangbin Hospital in ZhenJiang. They were 52-85 years old, without Alzheimer's disease or other diseases diagnosed by physical examination.

\section{Methods}

\section{Immunohistochemistry}

Blood was collected into tubes containing sodium citrate. Leukocytes were then isolated by lysing RBC with BD Pharm LyseTM lysing buffer (BD Biosciences, USA), followed by centrifugation at 2,000 rpm for 5 
min. After a series of washes in PBS, cells were resuspended in PBS. Cells were then applied onto poly-Llysine coated glass slides, which were air dried and stored at room temperature until use. For IHC slides were washed with PBS and fixed for 15 min with 3.7\% paraformaldehyde in PBS. After sequential rinsing with PBS and PBS $+0.5 \%$ Triton X-100, and blocking with BSA blocking buffer, slides were incubated overnight in a humid chamber at $40^{\circ} \mathrm{C}$ with rabbit polyclonal primary antibodies targeted at the $\mathrm{N}$-terminus of APP (1:100, Abgent, USA). After washing, bound primary antibodies were detected either by FITC-conjugated (Sigma) or AP-conjugated (Jackson) secondary antibodies. The BCIP/NBT kit was used in alkaline phosphatase (AP)-based immunoblotting detection. Images were acquired on an Olympus IX71 microscope.

For statistical analysis, t-test was used to compare the expression of APP in different cell types between AD patients and non-dementia controls.

\section{Flow cytometry}

APP protein expression levels in peripheral blood cells were analyzed precisely using flow cytometry. $100 \mu \mathrm{L}$ of peripheral blood from Alzheimer's disease and control patients, respectively, were assessed. After red blood cell lysis with BD Pharm LyseTM lysing buffer (BD Biosciences, USA), leukocytes were treated with $2 \%$ paraformalde-hyde, and permeabilized with $90 \%$ cold methanol. Primary antibody specific to an APP aminoterminal epitope (Abgent, USA) and FITC-conjugated secondary antibodies (Sigma) were used. Antibodybound cells were finally re-suspended in $1 \mathrm{~mL}$ PBS. The fluorescence of 10,000 cells was measured using FACS Calibur (BD Bioscience, USA). FITC green fluorescence (APP signal) was analyzed within the context of a forward scatter (FSC) and side scatter (SSC) gate established to include granulocytes, monocytes and lymphocytes. Fluorescence intensity data were expressed as geometrical mean (GEO mean) for each cell type.

\section{Results}

Higher APP expression in some peripheral blood leukocytes from Alzheimer's disease patients

Leukocyte APP protein expression in samples from Alzheimer's disease patients and controls was assessed by fluorescence inverted microscope after immunohistochemical staining (Figure 1, 400x magnification). Figure $1 \mathrm{~A}$ and $\mathrm{B}$ show immunofluorescence data, while Figure $1 \mathrm{C}$ and D depict alkaline phosphatase staining results. Although basal expression of the APP protein was found in the control group, the intensity of APP signals was higher in some cell types from Alzheimer's disease patient samples. Interestingly, APP was mainly expressed in the cell membrane and/or cytoplasm for most positive cells, with the cellular expression often

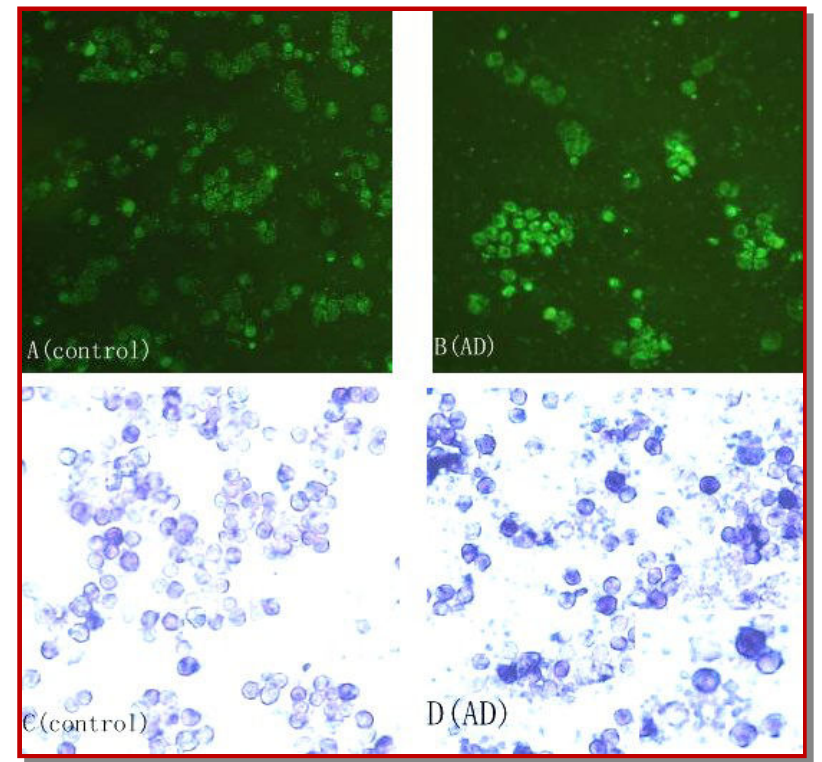

Figure 1: Immunohistochemistry detection of leukocyte APP. Panel A and B, immunofluorescence staining; Panel C and D, alkaline phosphatase staining

not evenly distributed.

Granulocytes from Alzheimer's disease patients express significantly higher amounts of APP compared with control values

Flow cytometry was used to assess APP expression in peripheral blood leukocytes. Blood cells from AD patients and healthy individuals $(n=9)$ were assessed by flow cytometry after fluorescence labeling. Figure $2 \mathrm{~A}$ shows the clustering of various types of peripheral blood cells. APP expression levels in AD patients and controls were detected and compared for lymphocytes, monocytes, and granulocytes (Figure 2). Interestingly, APP expression levels in lymphocytes, monocytes, and neutrophils from Alzheimer's disease patients were all higher compared with control values (Figure 2B and C).

Independent sample $\mathrm{t}$-test (Table I) was conducted to compare the Alzheimer's disease and control groups, with $95 \%$ confidence intervals determined. $T$ values of $1.045(p=0.312)$ and $1.264(p=0.224)$ were obtained for lymphocytes and monocytes, suggesting a higher APP expression in lymphocytes and monocytes from patients with Alzheimer's disease compared with control values, but the differences were not statistically significant. Meanwhile, a T value of $2.418(p=0.03)$ was obtained for granulocytes, indicating significantly higher APP expression in granulocytes from Alzheimer's disease patients compared with control values.

\section{Discussion}

Current clinical diagnosis of Alzheimer's disease requires intensive examination that includes neuropsycho- 


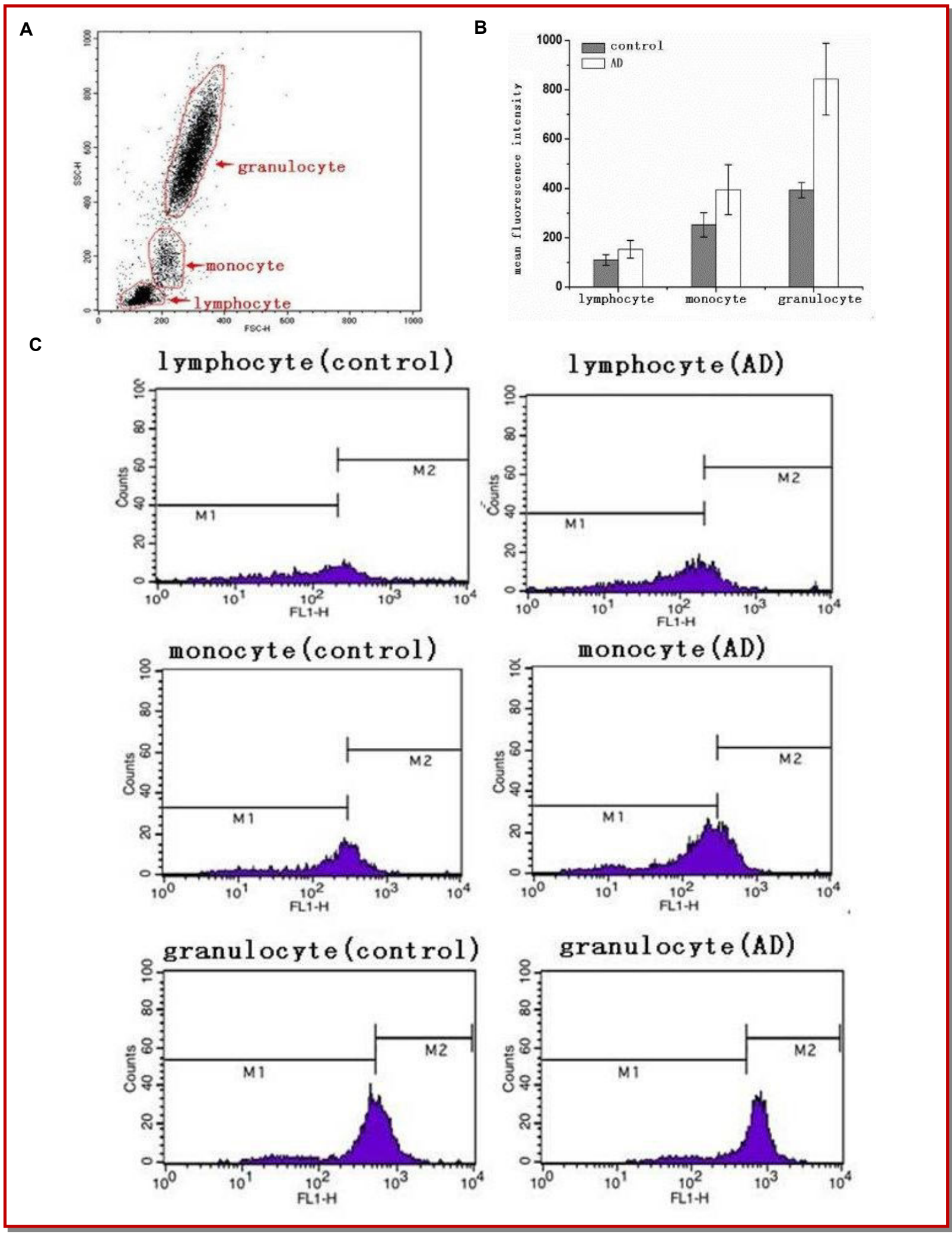

Figure 2: APP expression in circulating leukocytes determined by flow cytometry. (A) Dot plot of cells examined ex vivo in whole blood. Granulocytes, monocytes, and lymphocytes were gated; (B) histograms of APP levels in lymphocytes, monocytes, and granulocytes, respectively, from Alzheimer's disease and control subjects; (C) bar chart of fluorescence intensity analysis in lymphocytes, monocytes, and granulocytes 
Table I

Fluorescence intensity (geometric mean) in lymphocytes, monocytes, and granulocytes

\begin{tabular}{|lccrr|r|}
\hline Sample & $\mathrm{n}$ & Lymphocytes & Monocytes & Granulocytes \\
\hline Control & 9 & $109.8 \pm 21.6$ & $252.4 \pm 48.9$ & $393.1 \pm 31.2$ \\
Alzheimer's disease & 9 & $153.4 \pm 35.8$ & $395.0 \pm 101.6$ & $843.1 \pm 145.9$ \\
\hline
\end{tabular}

Data are mean $\pm \mathrm{SD}$

logical testing and costly brain imaging techniques, and a definitive diagnosis can only be made upon postmortem neuropathological evaluation. Additionally, ante-mortem clinical Alzheimer's disease diagnosis is typically administered following onset of cognitive and behavioral symptoms. As these symptoms emerge relatively late in the disease course, therapeutic intervention generally occurs after significant neurodegeneration, with limited efficacy. Therefore, identification of noninvasive diagnostic biomarkers of Alzheimer's disease is becoming increasingly important; they would provide diagnosis tools to clinics with limited access to neuropsychological testing or state of the art brain imaging, reduce clinical diagnosis cost, constitute biological parameters to track the course of therapeutic interventions, and most importantly, allow for earlier diagnosis possibly even during the prodromal phase, with hopes of therapeutic intervention prior to appreciable neurodegeneration.

In this study, APP expression levels in different peripheral blood leukocyte types were assessed by flow cytometry, a highly sensitive method. The results obtained by flow cytometry and immunohistochemistry were consistent. By analyzing APP expression in peripheral blood leukocytes, including lymphocytes, monocytes, and granulocytes, high APP levels were found in some cells, especially in the membrane and/or cytoplasm. Statistical analysis suggested that APP expression in granulocytes from Alzheimer's disease patients is significantly higher compared with values obtained in healthy individuals; however, no statistically significant differences were observed for lymphocytes and mononuclear cells.

Magaki et al. (2008) reported increased APP expression on lymphocyte or monocyte surface in MCI subjects compared with controls, with no differences observed in granulocytes (Magaki et al., 2008). Here, surface as well as intracellular APP levels in lymphocytes, monocytes, and granulocytes were measured. The increased APP expression in granulocytes is probably due to the higher intracellular APP production. The changes of methylation patterns and expression of multiple genes in Alzheimer's disease patient leukocytes (Hou et al., 2013) might explain the change of APP expression in leukocytes.

Platelets, considered a major source of peripheral APP, can generate the different APP forms via cleavage by aand $\beta$ - and other secretases, followed by storage in platelet a granules. When platelets are activated, various APP isomers and $A \beta$ are released, some of which penetrate through the compromised blood brain barrier and are deposited in the extracellular space in the brain, leading to senile plaque generation (Di Luca et al., 2000; $\mathrm{Li}$ et al., 1998). As shown above, granulocytes, lymphocytes and monocytes from peripheral blood cells all express the APP protein, and its expression levels are significantly higher in samples from Alzheimer's disease patients than those from control subjects. It has been suggested that Alzheimer's disease is associated with granulocyte density (Jaremo et al., 2013). In addition, ineffective phagocytosis of amyloid-beta by macrophages/monocytes in Alzheimer's disease patients was reported (Fiala et al., 2005). Furthermore, the phagocytic activity of peripheral neutrophils is altered in Alzheimer's disease patients. Neutrophils were shown to retain the ability to engulf microbes, but their digestive activity decreased at the early stage of Alzheimer's disease (Davydova et al., 2003). We speculate that APP might be engulfed and digested by peripheral neutrophils/PMN leukocytes, when circulating at high levels under disease state. Therefore, decreased digestive activity of APP in PMN leukocytes might lead to the elevated APP levels observed in granulocytes from Alzheimer's disease patients. Our results indicate a potential close correlation between the APP accumulation in peripheral PMN leukocytes and Alzheimer's disease pathogenesis, which may help further uncover the physiological and biochemical mechanism of Alzheimer's disease; this merits further study.

\section{Conclusion}

APP levels in peripheral blood granulocytes may be used as attractive Alzheimer's disease biomarker candidate for early diagnosis, as peripheral blood cells can be obtained in a minimally invasive manner and are easily analyzed by widely available cytometry techniques.

\section{Acknowledgement}

This work was supported by the National Natural Science Foundation of China (31272508). 


\section{References}

Avila J, Perez M, Lucas JJ, Gomez-Ramos A, Santa Maria I, Moreno F, Smith M, Perry G, Hernandez F. Assembly in vitro of tau protein and its implications in Alzheimer's disease. Curr Alzheimer Res. 2004; 1: 97-101.

Blennow K. CSF biomarkers for Alzheimer's disease: Use in early diagnosis and evaluation of drug treatment. Expert Rev Mol Diagn. 2005; 5: 661-72.

Blennow K, Hampel H, Weiner M, Zetterberg H. Cerebrospinal fluid and plasma biomarkers in Alzheimer disease. Nat Rev Neurol. 2010; 6: 131-44

Borroni B, Colciaghi F, Corsini P, Akkawi N, Rozzini L, Del Zotto E, Talarico G, Cattabeni F, Lenzi GL, Di Luca M, Padovani A. Early stages of probable Alzheimer disease are associated with changes in platelet amyloid precursor protein forms. Neurol Sci. 2002; 23: 207-10.

Davydova TV, Fomina VG, Voskresenskaya NI, Doronina OA. Phagocytic activity and state of bactericidal systems in polymorphonuclear leukocytes from patients with Alzheimer's disease. Bull Exp Biol Med. 2003; 136: 355-57.

Di Luca M, Colciaghi F, Pastorino L, Borroni B, Padovani A, Cattabeni F. Platelets as a peripheral district where to study pathogenetic mechanisms of alzheimer disease: The case of amyloid precursor protein. Eur J Pharmacol. 2000; 405: 27783.

Dulubova I, Ho A, Huryeva I, Sudhof TC, Rizo J. Threedimensional structure of an independently folded extracellular domain of human amyloid-beta precursor protein. Biochemistry 2004; 43: 9583-88.

Evin G, Li QX. Platelets and Alzheimer's disease: Potential of APP as a biomarker. World J Psychiatr. 2012; 2: 102-13.

Fiala M, Lin J, Ringman J, Kermani-Arab V, Tsao G, Patel A, Lossinsky AS, Graves MC, Gustavson A, Sayre J, Sofroni E, Suarez T, Chiappelli F, Bernard G. Ineffective phagocytosis of amyloid-beta by macrophages of Alzheimer's disease patients. J Alzheimers Dis. 2005; 7: 221-32.

Gustaw-Rothenberg KA, Siedlak SL, Bonda DJ, Lerner A Tabaton M, Perry G, Smith MA. Dissociated amyloid-beta antibody levels as a serum biomarker for the progression of Alzheimer's disease: A population-based study. Exp Gerontol. 2010; 45: 47-52.

Hardy J, Selkoe DJ. The amyloid hypothesis of Alzheimer's disease: Progress and problems on the road to therapeutics. Science 2002; 297: 353-56.

Hou Y, Chen H, He Q, Jiang W, Luo T, Duan J, Mu N, He Y, Wang $\mathrm{H}$. Changes in methylation patterns of multiple genes from peripheral blood leucocytes of Alzheimer's disease patients. Acta Neuropsychiatr. 2013; 25: 66-76.

Jaremo P, Milovanovic M, Buller C, Nilsson S, Winblad B. Alzheimer's disease and granulocyte density diversity. Eur J
Clin Invest. 2013; 43: 545-48.

Jiang S, Zhang M, Ren D, Tang G, Lin S, Qian Y, Zhang Y, Jiang K, Li F, Wang D. Enhanced production of amyloid precursor protein mRNA by peripheral mononuclear blood cell in Alzheimer's disease. Am J Med Genet B Neuropsychiatr Genet. 2003; 118b: 99-102.

Kawahara M. Disruption of calcium homeostasis in the pathogenesis of Alzheimer's disease and other conformational diseases. Curr Alzheimer Res. 2004; 1: 87-95.

Li QX, Fuller SJ, Beyreuther K, Masters CL. The amyloid precursor protein of Alzheimer disease in human brain and blood. J Leukoc Biol. 1999; 66: 567-74.

Li QX, Whyte S, Tanner JE, Evin G, Beyreuther K, Masters CL. Secretion of Alzheimer's disease Abeta amyloid peptide by activated human platelets. Lab Invest. 1998; 78: 461-69.

Magaki S, Yellon SM, Mueller C, Kirsch WM. Immunophenotypes in the circulation of patients with mild cognitive impairment. J Psychiatr Res. 2008; 42: 240-46.

Mangialasche F, Westman E, Kivipelto M, Muehlboeck JS, Cecchetti R, Baglioni M, Tarducci R, Gobbi G, Floridi P, Soininen H, Kloszewska I, Tsolaki M, Vellas B, Spenger C, Lovestone S, Wahlund LO, Simmons A, Mecocci P. Classification and prediction of clinical diagnosis of Alzheimer's disease based on MRI and plasma measures of alpha-/ gamma-tocotrienols and gamma-tocopherol. J Intern Med. 2013; 273: 602-21.

Marksteiner J, Hinterhuber H, Humpel C. Cerebrospinal fluid biomarkers for diagnosis of Alzheimer's disease: Beta-amyloid (1-42), tau, phospho-tau-181 and total protein. Drugs Today (Barc). 2007; 43: 423-31.

Mukaetova-Ladinska EB, Abdel-All Z, Dodds S, Andrade J, Alves da Silva J, Kalaria RN, O'Brien JT. Platelet immunoglobulin and amyloid precursor protein as potential peripheral biomarkers for Alzheimer's disease: Findings from a pilot study. Age Ageing. 2012; 41: 408-12.

Padovani A, Borroni B, Colciaghi F, Pettenati C, Cottini E, Agosti C, Lenzi GL, Caltagirone C, Trabucchi M, Cattabeni F, Di Luca M. Abnormalities in the pattern of platelet amyloid precursor protein forms in patients with mild cognitive impairment and Alzheimer disease. Arch Neurol. 2002; 59: 71-75.

Ratnavalli E, Brayne C, Dawson K, Hodges JR. The prevalence of frontotemporal dementia. Neurology 2002; 58: 1615-21.

Shad KF, Aghazadeh Y, Ahmad S, Kress B. Peripheral markers of Alzheimer's disease: Surveillance of white blood cells. Synapse 2013; 67: 541-43.

Tabaton M, Piccini A. Role of water-soluble amyloid-beta in the pathogenesis of Alzheimer's disease. Int J Exp Pathol. 2005; 86: 139-45.

Tortosa A, Lopez E, Ferrer I. Bcl-2 and Bax protein expression in Alzheimer's disease. Acta Neuropathol. 1998; 95: 407-12. 


\section{Your feedback about this paper}

1. Number of times you have read this paper 0

2. Quality of paper
Excellent
Good
Moderate
Not good

3. Your comments 